\title{
PRIMER ESTUDIO PSICOMÉTRICO DE LA VERSIÓN ESPAÑOLA DEL AGGRESSIVE SEXUAL BEHAVIOR INVENTORY (ASBI)
}

\author{
JUAN C. SIERRA ${ }^{1}$, José R. GUTIÉRREZ-QuintANILLA ${ }^{2}$ \\ y CARlos J. DELGADO-DOMÍNGUEZ ${ }^{1}$ \\ ${ }^{1}$ Facultad de Psicología, Universidad de Granada, España \\ ${ }^{2}$ Universidad Tecnológica, El Salvador
}

\begin{abstract}
Resumen: Este estudio instrumental analiza la estructura interna, fiabilidad y validez del Aggressive Sexual Behavior Inventory (ASBI), proporcionando los primeros datos psicométricos en muestras hispanas. Para ello, el ASBI, el STAXI-2, el Cuestionario de Agresión de Buss y Perry, y la Escala de Deseabilidad Social de Crowne y Marlowe fueron administrados a dos muestras formadas por 227 y 699 hombres, respectivamente, todos ellos estudiantes universitarios salvadoreños. Los resultados encontrados no dan apoyo a la estructura multidimensional planteada originalmente por los autores del ASBI, siendo más recomendable el uso de una puntuación total. Esta estructura unidimensional presenta valores alfa de Cronbach de 0,90 y 0,85 en cada una de las muestras. Se evidencia también que esta versión española del ASBI muestra una adecuada validez convergente y divergente. Por último, se discute la influencia de la deseabilidad social en la evaluación de la conducta sexual agresiva en el hombre.
\end{abstract}

Palabras clave: ASBI, conducta sexual agresiva, fiabilidad, validez.

First psychometric study of the Spanish version of the Aggressive Sexual Behavior Inventory (ASBI)

\begin{abstract}
This instrumental study analyzes the internal structure, reliability and the validity of the Aggressive Sexual Behavior Inventory (ASBI) and provides the first psychometric data of Hispanic samples. For this purpose, the ASBI, the STAXI - 2, the Buss-Perry Aggression Questionnaire and the Crowne-Marlowe Social Desirability Scale were applied in two samples consisting of respectively 227 and 699 men, all of them Salvadorian university students. The results do not support the multidimensional structure which was proposed originally by the authors of the ASBI, being the usage of total scores more recommended. This one-dimensional structure presents Cronbach alpha values of 0.90 and 0.85 in each sample. Evidence was found that this Spanish version of the ASBI has an adequate convergent and divergent validity. Finally, the influence of social desirability in the evaluation of aggressive sexual behaviour in men is discussed.
\end{abstract}

Keywords: ASBI, aggressive sexual behavior, reliability, validity.

\section{INTRODUCCIÓN}

Dentro de la categoría de violencia interpersonal, la violencia conyugal constituye uno de los problemas sociales y de salud pública que mayor atención está adquiriendo en la actualidad. A pesar de que ambos miembros de una pareja pueden ser víctimas de este problema, en

Recibido 12 junio 2007; aceptado 26 octubre 2007

Correspondencia: Juan Carlos Sierra, Facultad de Psicología. Universidad de Granada, 18071 Granada, España. Correo-e: jcsierra@ugr.es la mayoría de las ocasiones es la mujer la que soporta los abusos, así como las importantes repercusiones psicopatológicas que les acompañan (Amor, Echeburúa, Corral, Sarasua y Zubizarreta, 2001). Se trata de un fenómeno de gran magnitud que puede afectar a todo tipo de mujeres, con independencia de su cultura, clase social o nivel económico. En función del tipo

Este estudio psicométrico forma parte de un Proyecto de Cooperación Universitaria financiado por la Consejería de la Presidencia de la Junta de Andalucía (España) (Expediente AI33/04) concedido al primer autor. 
de conductas manifestadas por el agresor es posible distinguir tres modalidades de violencia dentro de la pareja: agresión física, agresión psicológica y agresión sexual (Labrador, Paz, De Luis y Fernández-Velasco, 2004; Ruiz-Pérez, Mata-Pariente y Plazaola-Castaño, 2006). La agresión sexual, aun compartiendo características del maltrato físico, se tiende a considerar de forma diferenciada debido a sus especiales connotaciones: por un lado, la intimidad asociada a este tipo de comportamientos y, por otro, que es frecuente considerar un «deber» $\mathrm{u}$ 《obligación» llevar a cabo con la pareja comportamientos sexuales, lo que en ocasiones provoca que las mujeres no sean conscientes de ser víctimas de violencia sexual por parte de sus parejas. La agresión sexual puede manifestarse bajo varias formas, tales como relaciones sexuales forzadas en el matrimonio o en una cita amorosa, relaciones sexuales no deseadas pero aceptadas como resultado de la insistencia, chantaje o amenaza, violación por un extraño y, en el peor de los casos, violación con consecuencia de muerte. Se estima que una de cada cuatro mujeres es sometida a algún acto de violencia sexual por un compañero íntimo en el transcurso de su vida (Jewkes, Sen y García-Moreno, 2002) y que el tiempo de convivencia suele ser un factor de riesgo (Cáceres y Cáceres, 2006). Este tipo de conductas agresivas tiene lugar tanto en países desarrollados, como por ejemplo España, donde durante el año 2006 se contabilizaron cerca de siete mil delitos de abuso, acoso y agresión sexual (Instituto de la Mujer, 2007b) y más de mil mujeres fueron víctimas de delitos contra la libertad e indemnidad sexual en el ámbito familiar (Instituto de la Mujer, 2007a), como en países en vías de desarrollo, como El Salvador, donde los índices de violencia hacia las mujeres alcanza cotas muy elevadas (Aron, Corne, Furslnad y Zelwer, 1991; Barthauer y Leventhal, 1999; Observatorio Centroamericano sobre Violencia, 2007; Orantes, 2004).

Abordar el estudio de la violencia sexual contra las mujeres requiere el uso de instrumentos de evaluación que presenten adecuadas garantías psicométricas. En las tres últimas décadas se han elaborado diversas medidas de autoinforme para la evaluación de las conductas sexuales agresivas, entre las cuales se encuentra el Aggressive Sexual Behavior Inventory (ASBI) de Mosher y Anderson (1986). Este instrumento fue desarrollado para evaluar las agresiones sexuales de los hombres hacia las mujeres que pueden tener lugar en citas o situaciones heterosexuales. Originalmente, sus autores plantearon una estructura multidimensional aislando en una muestra de estudiantes universitarios $(N=175)$ hasta seis factores diferentes cuyos valores de consistencia interna oscilaron entre 0,73 y 0,83 : Fuerza sexual $(\alpha=$ $0,83)$, Drogas y alcohol $(\alpha=0,81)$, Manipulación verbal $(\alpha=0,77)$, Rechazo con enfado $(\alpha$ $=0,79)$, Expresión de cólera $(\alpha=0,73)$ y Amenazas $(\alpha=0,76)$. Además, Mosher y Anderson (1986) informaron de un elevado índice de consistencia interna para la escala total $(\alpha=0,94)$, lo que sugiere la homogeneidad de los 20 ítems que la conforman. Por lo que respecta a su validez, se encontraron correlaciones significativas entre la puntuación total del ASBI y personalidad machista $(r=0,33)$, actitudes sexuales crueles $(r=0,53)$ y violencia machista $(r=$ 0,23) (Mosher y Anderson, 1986).

El objetivo de este estudio, catalogado como instrumental según la clasificación de Montero y León (2007) y para cuya elaboración se tuvieron en cuenta las recomendaciones de Carretero-Dios y Pérez (2007), fue llevar a cabo una adaptación del ASBI a población salvadoreña, para lo cual aplicamos una versión traducida del mismo a dos muestras de estudiantes universitarios, aportando información sobre su estructura factorial, fiabilidad y validez.

\section{MÉTODO}

\section{Muestras}

Se emplearon dos muestras de hombres, todos ellos estudiantes universitarios seleccionados mediante procedimiento no aleatorio. $\mathrm{La}$ primera muestra estaba formada por 227 estudiantes de la Universidad Tecnológica de El Salvador (media de edad de 23,96 años; $D T=4,77)$. La segunda muestra la componían 699 estudiantes de seis universidades de San Salvador: Universidad Tecnológica de El Salvador (23,90\%), Universidad Francisco Gavidia $(21,30 \%)$, Universidad Centroamericana 
José Simeón Cañas (21,20\%), Universidad Modular Abierta (10\%), Universidad Don Bosco $(14,90 \%)$ y Universidad Evangélica $(8,70 \%)$; la media de edad de esta segunda muestra era de 21,89 años $(D T=4,10)$.

\section{Instrumentos}

Aggressive Sexual Behavior Inventory (ASBI) (Mosher y Anderson, 1986). Está compuesto por 20 ítems contestados en escala tipo Likert desde 1 (nunca) a 7 (siempre), por lo que la puntuación total oscila entre 20 y 140, indicando las puntuaciones elevadas una mayor frecuencia de conductas sexuales agresivas. El Anexo 1 recoge la versión utilizada en esta investigación.

Escala de Deseabilidad Social (Crowne y Marlowe, 1960). Se empleó la adaptación española de Ferrando y Chico (2000) compuesta por 33 ítems con un formato de respuesta dicotómico (verdadero-falso). Diferentes estudios informan de valores para el coeficiente alfa que oscilan entre 0,75 y 0,85 (Ballard, 1992; Borkenau y Ostendorf, 1992; Reynolds, 1982; Strahan y Gerbasi, 1972), aunque tal como indica Collazo (2005) su estructura factorial no está excesivamente clara. En este estudio se obtuvo para la escala total un coeficiente de consistencia interna de 0,72 en la primera muestra y de 0,74 en la segunda.

Inventario de Expresión de Ira Estado-Rasgo, STAXI-2. Se empleó la versión española de Miguel-Tobal, Casado, Cano-Vindel y Spielberger (2001) formada por 49 ítems agrupados en seis escalas: Estado de ira, Rasgo de ira, Expresión externa de ira, Expresión interna de ira, Control Externo de ira y Control interno de ira; la fiabilidad osciló entre 0,67 de Expresión interna de ira y 0,89 de Estado de ira. En el presente estudio, se utilizaron cuatro dimensiones: Ira estado, Ira rasgo, Expresión de ira (combinación de expresión externa e interna) y Control de ira (combinación de control externo e interno), obteniéndose valores para el coeficiente alfa de Cronbach entre 0,74 (Expresión de ira) y 0,90 (Estado de ira).

Cuestionario de Agresión de Buss y Perry (1992). La versión original está compuesta por
29 ítems a los que se le da respuesta en una escala tipo Likert de cinco puntos desde 1 (completamente falso para mi) hasta 5 (completamente verdadero para mi). En cuanto a la dimensionalidad, sus autores aíslan cuatro subescalas (Agresividad fisica, Agresividad verbal, Ira y Hostilidad), situándose sus valores de consistencia interna entre 0,72 y 0,85 . En este estudio se empleó la versión salvadoreña de Sierra y Gutiérrez-Quintanilla (2007), compuesta por 27 ítems que conforman los cuatro factores planteados en la versión original, obteniéndose valores para el coeficiente alfa entre 0,73 y 0,80 , así como correlaciones significativas con las dimensiones del STAXI-2.

\section{Procedimiento}

En la elaboración de la versión española del $A S B I$ se siguieron los siguientes pasos. En primer lugar, se realizó la traducción de los ítems al castellano; para esta tarea se seleccionaron tres profesores expertos del Departamento de Idioma de la Universidad Tecnológica de El Salvador (UTEC); dos de los profesores se encargaron de traducir el instrumento del inglés al español y el tercero revisó y homogeneizó las diferencias lingüísticas encontradas en la redacción de los ítems traducidos, de tal forma que no se perdiera el sentido original de los mismos, alcanzándose un total acuerdo entre los tres especialistas. A continuación, se envió la versión traducida a diez profesores de Psicología (salvadoreños y españoles), quienes tenían que indicar si estaban o no de acuerdo con la redacción de cada uno de los ítems, y redactar de forma correcta aquellos ítems con los que mostrasen discrepancia. Se obtuvieron los porcentajes de acuerdo y desacuerdo de cada ítem, alcanzándose en todos los elementos un porcentaje de acuerdo entre los expertos próximo al 100\%. Con el fin de prevenir posibles dificultades en la comprensión de los ítems de la nueva versión del instrumento, se llevó a cabo un ensayo piloto en 26 estudiantes de Psicología de la UTEC, proporcionándoles unas breves instrucciones sobre el objetivo de dicha aplicación: señalar el acuerdo o desacuerdo en 
cuanto a la comprensión de cada ítem, indicando aquello que no comprendían; en todos los ítems se alcanzó un porcentaje de acuerdo superior al 90\%. La versión definitiva del ASBI, junto con los otros tres instrumentos, fue aplicada por dos investigadores en las aulas de los distintos centros docentes participantes. La participación fue completamente voluntaria y ningún sujeto rehusó contestar a los cuestionarios. A todos ellos se les informó acerca del anonimato y confidencialidad de sus respuestas. Se aplicó la Escala de Deseabilidad Social con el fin de controlar posibles sesgos en las respuestas de los participantes.

\section{RESULTADOS}

\section{Análisis de items}

En primer lugar, se llevó a cabo un análisis de ítems y de la fiabilidad de la escala, manteniendo la estructura propuesta inicialmente por Mosher y Anderson (1986) (véase la Tabla 1).

El primer aspecto que llama la atención son los bajos valores de las medias de los diferentes ítems, oscilando entre 1,10 y 2,45 , lo cual está reflejando que las conductas sexuales agresivas que incluye el $A S B I$ tienen una baja incidencia entre la muestra evaluada. Los ítems de la primera dimensión (Fuerza sexual) presentaron valores medios de respuesta entre 1,10 del ítem 11 (He tranquilizado a una mujer con una o dos buenas bofetadas al ponerse histérica con mis forcejeos) y 1,37 del ítem 3 (He esperado mi turno entre otros hombres que estaban compartiendo a una "chica de la vida alegre"); las desviaciones típicas fueron inferiores a 1 , a excepción del ítem 3 , oscilando entre 0,50 (ítem 11) y 0,89 (ítem 9). La correlación de cada ítem con el resto de la escala mostró valores superiores en todos los casos a 0,40 . La consistencia interna fue 0,80 , aunque la eliminación del ítem 3 incrementaría ligeramente este valor. En cuanto a la segunda dimensión, Drogas y alcohol, las respuestas a sus ítems mostraron valores medios entre 1,20 del ítem 15 (Le he dado una droga cara a una mujer para que se sintiera obligada a hacerme un favor sexual) y 1,48 del ítem 2 (He emborrachado a una mujer para tener sexo con ella); las desviaciones típicas se aproximaron a 1 . Los valores de la correlación ítem-total fueron superiores a 0,40 y la consistencia interna 0,73 , incrementándose de forma considerable hasta 0,84 con la eliminación del ítem 2. En la tercera dimensión (Manipulación verbal), los valores medios de respuesta fueron algo más elevados, oscilando entre 1,61 del ítem 20 (Le he dicho a una mujer que su rechazo a tener sexo conmigo estaba cambiando mis sentimientos hacia ella) y 2,45 del ítem 4 (Le he dicho a una mujer que he querido entrar a su departamento y así poder hacerle el amor donde yo quisiera); las desviaciones típicas oscilaron entre 1,29 (ítem 20) y 1,99 (ítem 4). Las correlaciones de cada ítem con el resto de la escala superaron el valor de 0,40 ; la consistencia interna de esta dimensión alcanzó un valor de 0,65. En los dos ítems de la cuarta dimensión (Rechazo con enfado) se obtuvieron valores medios de respuesta también bajos $(1,30$ y 1,45$)$ y desviaciones típicas en torno al 1. La correlación ítem-total se situó para ambos ítems en 0,39 y el valor del alfa de Cronbach para esta dimensión fue 0,55. En el factor Expresión de cólera, las puntuaciones medias de los ítems oscilaron entre 1,31 del ítem 18 (He tomado a una mujer fuertemente y la he visto muy enojado cuando no me respondia sexualmente como yo quería) y 1,50 del ítem 8 (He perdido los estribos y he quebrado algo para mostrarle a una mujer que no debía enojarme), siendo las desviaciones típicas superiores a 1; las correlaciones ítem-total corregidas se situaron por encima de 0,40 , obteniéndose un alfa de Cronbach de 0,66. En la sexta y última dimensión (Amenazas), los dos ítems que la conforman presentan una media de 1,47 y 1,27 , respectivamente, y desviaciones típicas en torno a uno; las correlaciones ítem-total corregidas se quedaron por debajo de 0,30 y el alfa de Cronbach fue de 0,38.

$\mathrm{La}$ insuficiente consistencia reflejada por los valores alfa de Cronbach en las dimensiones 3, 4, 5 y 6 de la propuesta original del $A S B I$ nos condujo a considerar otras posibilidades de la estructura de la escala, por lo que se llevó a cabo un análisis factorial exploratorio. 
Tabla 1. Media, desviación típica, correlación ítem-total y alfa de Cronbach si el ítem es eliminado para cada una de las seis dimensiones del ASBI planteadas por sus autores originales $(\mathrm{N}=227)$

\begin{tabular}{|c|c|c|c|c|}
\hline Factor 1. Fuerza sexual $(\alpha=0,80)$ & $M$ & $D T$ & $r I-T$ & $a$ \\
\hline $\begin{array}{l}\text { 3. He esperado mi turno entre otros hombres que estaban compartiendo a una «chica de la vida } \\
\text { alegre". }\end{array}$ & 1,37 & 1,08 & 0,43 & 0,82 \\
\hline 9. He traído a mi casa a una mujer después de una cita y la he obligado a tener sexo conmigo. & 1,27 & 0,89 & 0,67 & 0,74 \\
\hline $\begin{array}{l}\text { 11. He tranquilizado a una mujer con una o dos buenas bofetadas al ponerse histérica por mis } \\
\text { forcejeos. }\end{array}$ & 1,10 & 0,50 & 0,47 & 0,79 \\
\hline 14. He obligado a una mujer a tener sexo conmigo y con algunos amigos. & 1,16 & 0,66 & 0,74 & 0,74 \\
\hline 17. He acostado a la fuerza a una mujer y la he hecho desvestirse o he roto su ropa si no cooperaba. & 1,22 & 0,87 & 0,64 & 0,75 \\
\hline 19. Me he emborrachado un poco y he obligado a la mujer con quien estoy a tener sexo conmigo. & 1,25 & 0,87 & 0,56 & 0,77 \\
\hline
\end{tabular}

Factor 2. Drogas y alcohol $(\alpha=0,73)$

2. He emborrachado a una mujer para tener sexo con ella.

6. He drogado a una mujer con marihuana o pastillas para que pudiera resistirse menos a mis $\quad \begin{array}{lllll}1,27 & 0,89 & 0,64 & 0,56\end{array}$ forcejeos.

15. Le he dado una droga cara a una mujer para que se sintiera obligada a hacerme un favor $\quad \begin{array}{llll}1,20 & 0,79 & 0,66 & 0,57\end{array}$ sexual.

Factor 3. Manipulación verbal $(\alpha=0,65)$

1. He amenazado con abandonar o terminar una relación si una mujer no tenía sexo conmigo. $\quad \begin{array}{lllll}1,79 & 1,59 & 0,50 & 0,54\end{array}$

4. Le he dicho a una mujer que he querido entrar a su departamento y así poder hacerle el amor $\quad 2,45 \quad 1,99 \quad 0,41 \quad 0,61$ donde yo quisiera.

7. Le he dicho a una mujer que por haberla estado acariciando tanto, no me podía dejar excitado. $\quad 2,17 \quad 1,78 \quad 0,42 \quad 0,59$

20. Le he dicho a una mujer que su rechazo a tener sexo conmigo estaba cambiando mis senti- $\quad \begin{array}{llll}1,61 & 1,29 & 0,43 \quad 0,60\end{array}$ mientos hacia ella.

Factor 4. Rechazo con enfado $(\alpha=0,55)$

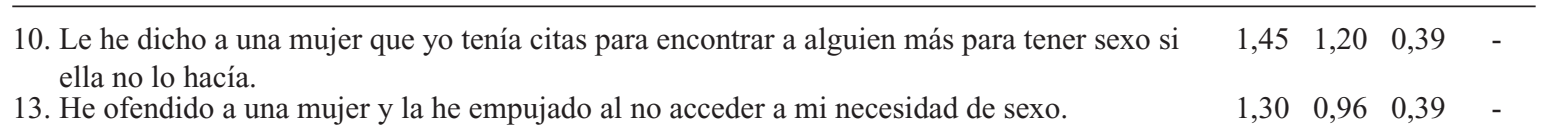

Factor 5. Expresión de cólera $(\alpha=0,66)$

8. He perdido los estribos y he quebrado algo para mostrarle a una mujer que no debía enojarme. $\quad 1,50 \quad 1,26 \quad 0,48 \quad 0,55$

16. He tratado mal a una mujer para que supiera que hablaba en serio.

$\begin{array}{llll}1,41 & 1,13 & 0,52 & 0,50\end{array}$

18. He tomado a una mujer fuertemente y la he visto muy enojado cuando no me respondía $\quad 1,31 \quad 1,04 \quad 0,42 \quad 0,63$ sexualmente como yo quería.

Factor 6. Amenazas $(\alpha=0,38)$

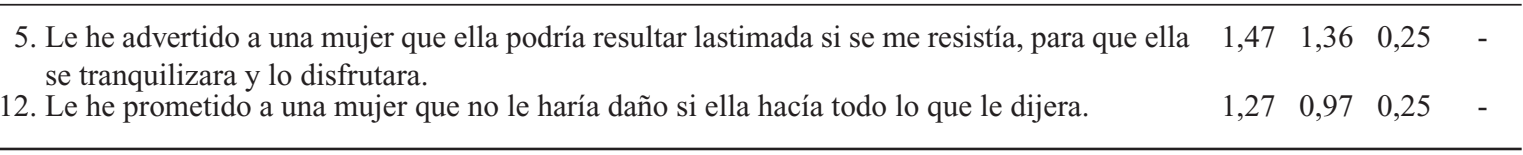

Nota: M: Media; DT : desviación típica; r I-T: correlación ítem-total; a: alfa de Cronbach si el ítem es eliminado. 


\section{Estructura factorial: análisis exploratorio}

Los resultados del test de Kaiser-MeyerOlkin $(\mathrm{KMO}=0,87)$ y la prueba de esfericidad de Barlett $\left(\chi^{2}{ }_{190}=2431,54 ; p<0,000\right)$ nos indican que los datos se adecuaban al empleo de esta técnica. El análisis factorial exploratorio, a través del método de extracción de componentes principales, prefijando seis factores, mostró una distribución de los ítems que difiere de forma sustancial de la propuesta original de los autores de la escala (véase la Tabla 2). Todos los ítems obtuvieron pesos factoriales superiores a 0,40 en un factor; no obstante, hay cuatro ítems $(5,16,19$ y 20$)$ que podrían ser asignados a más de un factor, pues la diferencia de cargas factoriales no alcanza una diferencia mínima de 0,10. El factor 1 agrupa a 13 de los 20 ítems $(14,15,18,6,17,13,11,12,9,19$, 5,20 y 16), provenientes de cada una de las seis dimensiones iniciales de Mosher y Anderson (1986); los valores de correlación ítem-total oscilaron entre 0,54 y 0,81 . El factor 2 reúne a los ítems 7, 1 y 4, los cuales formaban parte de la dimensión Manipulación verbal original; la correlación ítem-total se sitúo entre 0,39 y 0,40. El factor 3 agrupa tres ítems $(10,19$ y 5$)$ provenientes de las dimensiones originales Rechazo con enfado, Fuerza sexual y Amenazas, respectivamente; la correlación entre estos ítems y la escala total osciló entre 0,55 y 0,65 . El factor 4 quedó conformado por los ítems 5 y 3 , provenientes de las dimensiones Amenazas y Fuerza sexual de Mosher y Anderson; la correlación ítem-total fue de 0,42 en ambos casos. El factor 5 lo formaron los ítems 2 y 20 que provienen de las dimensiones originales Drogas y alcohol y Manipulación verbal, respectivamente, siendo la correlación ítem-total de ambos ítems de 0,45 . Por último, el factor 6 agrupó otros dos ítems (8 y 16), los cuales se situaban en la dimensión original Expresión de cólera; la correlación ítem-total fue de 0,47 en ambos ítems.

Tabla 2. Matriz de componentes principales con rotación Varimax prefijando seis factores: valores de saturación $(\mathrm{N}=227)$

\begin{tabular}{|c|c|c|c|c|c|c|c|}
\hline Item & Factor 1 & Factor 2 & Factor 3 & Factor 4 & Factor 5 & Factor & 6 \\
\hline 14 & 0,93 & & & & & & \\
\hline 15 & $\mathbf{0 , 8 7}$ & & & & & & \\
\hline 18 & 0,79 & & & & & & \\
\hline 6 & 0,79 & & & 0,35 & & & \\
\hline 17 & 0,78 & & & & & & \\
\hline 13 & 0,76 & & & & & & \\
\hline 11 & 0,70 & & & & & & \\
\hline 12 & 0,66 & 0,32 & & & & & \\
\hline 9 & 0,65 & & 0,34 & & & & \\
\hline 7 & & 0,76 & & & & & \\
\hline 1 & & 0,68 & & & & & \\
\hline 4 & & 0,63 & & & & & \\
\hline 10 & 0,35 & & 0,67 & & & 0,34 & \\
\hline 19 & 0,55 & & 0,60 & & 0,31 & & \\
\hline 5 & 0,45 & & 0,47 & 0,41 & & & \\
\hline 3 & 0,42 & & & 0,73 & & & \\
\hline 2 & 0,33 & & & & 0,73 & & \\
\hline 20 & $\mathbf{0 , 5 0}$ & & 0,43 & & 0,54 & & \\
\hline 8 & 0,46 & & & & & 0,72 & \\
\hline 16 & 0,55 & & & & & 0,57 & \\
\hline Alfa & 0,91 & 0,60 & 0,75 & 0,58 & 0,62 & 0,63 & \\
\hline$\%$ Varianza & & & & & & & \\
\hline explicada & 36,26 & 10,06 & 7,93 & 6,50 & 5,73 & 5,63 & \\
\hline Valor propio & 7,25 & 2,01 & 1,59 & 1,30 & 1,15 & 1,13 & \\
\hline
\end{tabular}

Nota: Se han omitido las cargas factoriales inferiores a 0,30 . 
Teniendo en cuenta que los valores de alfa de la mayoría de las dimensiones no alcanzaron valores satisfactorios (véase la Tabla 2), que la reagrupación de ítems resultante es difícilmente interpretable debido a la heterogeneidad del contenido de los ítems que conforman las diferentes dimensiones y que la mayoría de los ítems se agrupaban en torno a un único factor, se llevó a cabo un segundo análisis de componentes principales prefijando un solo factor. Este planteamiento se fundamenta en el hecho de que los autores de la escala original informaron de un elevado coeficiente de consistencia para la escala total $(\alpha=0,94)$ y de correlaciones de la escala total con otras variables criterio. Esta solución unifactorial explicó el $42,18 \%$ de la varianza total, presentando todos los ítems un peso factorial superior a 0,40 (véase la Tabla 3).

\section{Validación cruzada de la estructura unifactorial}

Con el objetivo de determinar si esta estructura unidimensional era estable se aplicó el $A S B I$ a una segunda muestra de 699 sujetos con características sociodemográficas similares a los de la primera. En esta ocasión, a diferencia de lo que ocurrió en la primera muestra, se encontró una correlación muy modesta, pero significativa, entre la puntuación de la Escala de Deseabilidad Social y el $A S B I(r=-0,10 ; p=0,01)$. Con el fin de controlar dicho sesgo, se eliminaron de los análisis aquellos sujetos que se situaban 1,5 desviaciones típicas o más por encima de la media en la Escala de Deseabilidad Social $(M=18,20 ; D T=5,03)$, tras lo cual la muestra pasó de 699 a 507 sujetos, dejando de ser significativa la correlación entre ambas variables $(r=-0,07 ; p=0,08)$. En esta ocasión, los valores del test de Kaiser-Meyer-Olkin (KMO $=0,89)$ y de la prueba de esfericidad de Bartlett $\left(\chi^{2}{ }_{190}=3965,676 ; p<0,000\right)$ también fueron adecuados para el empleo del análisis factorial. Tal como se aprecia en la Tabla 3, la varianza explicada en esta segunda muestra fue ligeramente menor, mientras que los pesos factoriales de los ítems presentaron valores bastante similares a los encontrados en la primera muestra, superando todos ellos el valor de 0,40 , situándose por debajo únicamente los ítems 4 y 7.

Tabla 3. Solución unifactorial: valores de saturación para análisis exploratorio I $(\mathrm{n}=227)$ y análisis exploratorio II $(\mathrm{n}=507)$

\begin{tabular}{|c|c|c|}
\hline Ítem & $\begin{array}{c}\text { Análisis } \\
\text { exploratorio I } \\
n=227\end{array}$ & $\begin{array}{c}\text { Análisis } \\
\text { exploratorio II } \\
n=507\end{array}$ \\
\hline 1 & 0,47 & 0,40 \\
\hline 2 & 0,54 & 0,40 \\
\hline 3 & 0,54 & 0,44 \\
\hline 4 & 0,41 & 0,37 \\
\hline 5 & 0,62 & 0,49 \\
\hline 6 & 0,77 & 0,64 \\
\hline 7 & 0,41 & 0,31 \\
\hline 8 & 0,60 & 0,51 \\
\hline 9 & 0,71 & 0,65 \\
\hline 10 & 0,59 & 0,76 \\
\hline 11 & 0,59 & 0,72 \\
\hline 12 & 0,62 & 0,60 \\
\hline 13 & 0,72 & 0,74 \\
\hline 14 & 0,82 & 0,65 \\
\hline 15 & 0,78 & 0,77 \\
\hline 16 & 0,64 & 0,56 \\
\hline 17 & 0,78 & 0,77 \\
\hline 18 & 0,77 & 0,64 \\
\hline 19 & 0,72 & 0,65 \\
\hline 20 & 0,68 & 0,52 \\
\hline Alfa Cronbach & 0,90 & 0,85 \\
\hline \% Varianza explicada & $42,18 \%$ & $35,56 \%$ \\
\hline Valor propio & 8,44 & 7,11 \\
\hline
\end{tabular}

Correlaciones entre las puntuaciones del ASBI y constructos afines

Con el objetivo de examinar la validez convergente y divergente de la versión española del $A S B I$ se correlacionó su puntuación total con una serie de constructos afines, hipotetizando una correlación positiva con ira estado, ira rasgo, expresión de ira, hostilidad, agresión física y agresión verbal, y una correlación negativa con control del ira. Tal como se puede apreciar en la Tabla 4, a pesar de que las correlaciones fueron muy modestas, todas ellas presentaron la dirección esperada, oscilando entre $-0,10$ (control de ira) y 0,29 (ira). 
Tabla 4. Correlaciones entre la puntuación total del ASBI y diferentes constructos afines $(\mathrm{N}=507)$

\begin{tabular}{lc}
\hline Constructos & $A S B I$ \\
\hline STAXI-2 & \\
Ira estado & $0,19^{* *}$ \\
Ira-rasgo & $0,16^{* *}$ \\
Expresión de ira & $0,16^{* *}$ \\
Control de ira & $-0,10^{*}$ \\
CA & \\
Hostilidad & $0,14^{* *}$ \\
Ira & $0,29^{* *}$ \\
Agresión física & $0,20^{* *}$ \\
Agresión verbal & $0,16^{* *}$ \\
\hline
\end{tabular}

Nota: ASBI: Aggressive Sexual Behavior Inventory; STAXI-2: Inventario de Expresión de Ira Estado-Rasgo; CA: Cuestionario de Agresión; ** $p<0,01 * p<0,05$.

\section{DISCUSIÓN}

Diversos estudios ponen de manifiesto que muchas mujeres son víctimas de la agresividad y coerción sexual por parte de los hombres (Barthauer y Leventhal, 1999; Instituto de la Mujer, 2007a, 2007b; Mills y Granoff, 1992; Observatorio Centroamericano sobre Violencia, 2007; Ruch y Wang, 2006; Sipsma, Carrobles Isabel, Montorio Cerrato y Everaerd, 2000; Ullman, Filipas, Townsend y Starzynski, 2006; Waszak Geary, Wedderburn, McCarraher, Cuthbertson y Pottinger, 2006). La evaluación de la conducta sexual agresiva del hombre hacia la mujer requiere de instrumentos que hayan demostrado adecuadas garantías psicométricas. Ante la escasez de autoinformes para evaluar este tipo de conductas en población hispana, nos propusimos analizar la estructura factorial, fiabilidad y validez de una versión española del Aggressive Sexual Behavior Inventory (ASBI) de Mosher y Anderson (1986).

En el estudio original, y a pesar del elevado coeficiente de consistencia interna que presentaba la escala total, se proponía por parte de sus autores una estructura multidimensional integrada por seis factores (Fuerza sexual, Drogas y Alcohol, Manipulación verbal, Rechazo con enfado, Expresión de cólera y Amenazas), obteniendo todos ellos índices de fiabilidad aceptables entre 0,73 y 0,83 . En cambio, nuestros resultados no son tan satisfactorios, presentándose falta de homogeneidad en la mayoría de las dimensiones propuestas originalmente por Mosher y Anderson (1986); así, excepto Fuerza sexual $(\alpha=0,80)$ y Drogas y alcohol $(\alpha=0,73)$, el resto de dimensiones se queda por debajo de 0,70 , que sería el valor mínimo de consistencia interna exigible, tal como señalan Carretero-Dios y Pérez (2007). El análisis de ítems pone de manifiesto que los valores promedio de respuesta oscilan entre 1,10 del ítem 11 (He tranquilizado a una mujer con una o dos buenas bofetadas al ponerse histérica por mis forcejeos) y 2,45 del ítem 4 (Le he dicho a una mujer que he querido entrar a su departamento y asi poder hacerle el amor donde yo quisiera), situándose muy por debajo del punto medio de la escala de respuesta $(3,5)$, lo que se justifica por el tipo muestra utilizada, la cual estaba conformada por estudiantes universitarios que supuestamente no presentan un perfil típico de agresor sexual.

Dada la baja homogeneidad que caracteriza a varios de los factores propuestos originalmente en la estructura factorial del ASBI, se llevó a cabo un análisis factorial exploratorio de componentes principales (rotación Varimax) prefijando seis factores, tal como plantearon en un primer momento Mosher y Anderson (1986). A pesar de que la solución factorial encontrada llegó a explicar un $72,11 \%$ de la varianza total, nuevamente la estructura interna de la escala difiere en gran medida de la versión original. La agrupación de ítems en las seis dimensiones no se ve respaldada por una coherencia teórica y conceptual de los mismos, encontrándose poca o nula relación entre los contenidos teóricos de los ítems que las conforman; además, a excepción de los factores $1(\alpha=0,91)$ y $3(\alpha=0,75)$, los cuatro restantes no alcanzan coeficientes adecuados de fiabilidad. Teniendo en cuenta la falta de apoyo encontrado para la estructura multidimensional de la versión española del ASBI, y considerando que en el estudio original la escala total había mostrado una adecuada homogeneidad $(\alpha=0,94)$, se llevó a cabo otro análisis factorial exploratorio prefijando en esta ocasión un solo factor. Esta nueva solución factorial explicó el $42,18 \%$ de la varianza total, presentando todos los ítems un peso factorial superior a 0,40 y un coeficiente de fiabilidad para la escala total de 0,90 , acercándose este valor al 
comunicado por Mosher y Anderson (1986). Esta estructura unidimensional fue ratificada en una segunda muestra, encontrándose valores muy similares a los hallados en la primera: $35,56 \%$ de varianza explicada, pesos factoriales superiores a 0,40 y fiabilidad de 0,85. Los resultados encontrados nos llevan a plantear que en la versión española del ASBI es más adecuado utilizar la puntuación total en lugar de las puntuaciones parciales de las dimensiones propuestas originalmente.

Con el objetivo de proporcionar indicios de validez a las medidas obtenidas con el ASBI se correlacionó su puntuación con constructos psicológicos afines como la ira, la hostilidad o la agresividad, dado que estudios previos (Malamuth, Linz, Heavey, Barnes y Acker, 1995; Malamuth, Sockloskie, Koss y Tanaka, 1991) habían puesto de manifiesto la asociación entre la coerción sexual y la hostilidad; además, modelos explicativos de violencia conyugal en los que se integrarían las agresiones sexuales, como el de Echeburúa y Fernández-Montalvo (1998), plantean que la hostilidad y el estado emocional de ira son factores determinantes en la explicación de dicho fenómeno. La conducta sexual agresiva correlacionó de forma positiva con ira (estado y rasgo), hostilidad y agresividad (física y verbal) (validez convergente) y de forma negativa con la puntuación en control de ira (validez divergente). A pesar de que las correlaciones son modestas, la dirección de las mismas pone de manifiesto que los individuos que realizan conductas sexuales coercitivas se caracterizan por presentar una personalidad hostil, tal como ya habían señalado Menard, Hall, Phung, Ghebrial y Martin (2003).

Una cuestión a tener en cuenta a la luz de los resultados encontrados es la influencia que la deseabilidad social tiene en las respuestas dadas al ASBI por un porcentaje importante de la muestra, teniendo que descartar de la segunda muestra del estudio el $27,47 \%$ de participantes. Consideramos que este sesgo debe ser tenido en cuenta y controlado en estudios de este tipo, pues probablemente este hecho no se trate de un fenómeno aislado, ya que también se ha puesto de manifiesto en investigaciones llevadas a cabo con estudiantes universitarios sobre doble moral sexual (Sierra y Gutiérrez-Quintanilla, en pren- sa) o actitudes favorables hacia la violación (Sierra, Gutiérrez-Quintanilla y Delgado-Domínguez, en prensa) y con población general sobre violencia marital (Sugarman y Hotaling, 1997).

En resumen, las medidas obtenidas con el ASBI en este primer estudio psicométrico en muestras hispanas parecen fiables y válidas, siendo más recomendable el uso de su puntuación total que el de las dimensiones propuestas por sus autores. En el futuro, el empleo de otras metodologías, como el análisis factorial confirmatorio, y de otras muestras deberán ratificar estos primeros resultados prometedores.

\section{REFERENCIAS}

Amor, P.J., Echeburúa, E., Corral, P., Sarasua, B., y Zubizarreta, I. (2001). Maltrato físico y maltrato psicológico en mujeres víctimas de violencia en el hogar: un estudio comparativo. Revista de Psicopatología y Psicología Clínica, 6, 167-178.

Aron, A., Corne, S., Fursland, A., y Zelwer, B. (1991). The gender-specific terror of El Salvador and Guatemala. Post-traumatic stress disorder in Central American refugee women. Women's Studies International Forum, 14, 37-47.

Ballard, R. (1992). Short forms of the Marlowe-Crowne social desirability scale. Psychological Reports, 71, 1155-1160.

Barthauer, L.M., y Leventhal, J.M. (1999). Prevalence and effects of child sexual abuse in a poor, rural community in El Salvador: A retrospective study of women after 12 years of civil war. Child Abuse \& Neglect, 23, 1117-1126.

Borkenau, P., y Ostendorf, F. (1992). Social desirability scales as moderator and suppressor variables. European Journal of Personality, 6, 199-214.

Buss, A. H., y Perry, M. (1992). The Aggression Questionnaire. Journal of Personality and Social Psychology, 63, 452-459.

Cáceres, A., y Cáceres, J. (2006). Violencia en relaciones íntimas en dos etapas evolutivas. International Journal of Clinical and Health Psychology, 6, 271-284.

Carretero-Dios, H., y Pérez, C. (2007). Standards for the development and review of instrumental studies: Considerations about test selection in psychological research. International Journal of Clinical and Health Psychology, 7, 863-882.

Collazo, A. (2005). Translation of the Marlowe-Crowne Social Desirability Scale into an equivalent Spanish version. Educational and Psychological Measurement, 65, 780-806. 
Crowne, D. P., y Marlowe, D. (1960). A new scale of social desirability independent of psychopathology. Journal of Consulting Psychology, 24, 349-354.

Echeburúa, E., y Fernández-Montalvo, J. (1998). Hombres maltratadores. Aspectos teóricos. En E. Echeburúa y P. Corral (Eds.), Manual de violencia familiar (pp.73-90). Madrid: Siglo XXI.

Ferrando, P. J., y Chico, E. (2000). Adaptación y análisis psicométrico de la escala de deseabilidad social de Marlowe y Crowne. Psicothema, 12, 383-389.

Instituto de la Mujer (2007a). Delitos conocidos contra la libertad e indemnidad sexual, según edad de la víctima y ámbito de comisión. Recuperado el 23 de marzo de 2007, de

http://www.mtas.es/mujer/mujeres/cifras/tablas/W869.xls

Instituto de la Mujer (2007b). Delitos conocidos de abuso, acoso y agresión sexual, por CCAA. Recuperado el 23 de marzo de 2007, de

http://www.mtas.es/mujer/mujeres/cifras/tablas/Wdelcc aa.XLS

Jewkes, R., Sen, P., y García-Moreno, C. (2002). La violencia sexual. Recuperado el 24 de Julio de 2006, de http://www.paho.org/Spanish/AM/PUB/capitulo_6.pdf

Labrador, F.J., Paz, P., De Luis, P., y Fernández-Velasco, R. (2004). Mujeres víctimas de violencia doméstica. Programa de actuación. Madrid: Pirámide

Malamuth, N.M., Linz, D., Heavey, C.L., Barnes, G., y Acker, M. (1995). Using the confluence model of sexual aggression to predict men's conflict with women: A 10-year follow-up study. Journal of Personality and Social Psychology, 69, 353-369.

Malamuth, N.M., Sockloskie, R.J., Koss, M.P., y Tanaka, J.S. (1991). Characteristics of aggresors against woemen: Testing a model using a national sample of college students. Journal of Consulting and Clinical Psychology, 59, 670-681.

Menard, K.S., Hall, G.C.N., Phung, A.H., Ghebrial, M.F.E., y Martin, L. (2003). Gender differences in sexual harassment and coercion in college students: Developmental, individual, and situational determinants. Journal of Interpersonal Violence, 18, 1222-1239.

Miguel-Tobal, J.J., Casado, M.I., Cano-Vindel, A., y Spielberger, C.D. (2001). Inventario de expresión de ira estado-rasgo. STAXI-2. Madrid: TEA.

Mills, C.S., y Granoff, B.J. (1992). Date and acquaintance rape among a sample of college students. Social Work, 37, 504-509.

Montero, I., y León, O.G. (2007). A guide for naming research studies in Psychology International Journal of Clinical and Health Psychology, 7, 847-862.

Mosher, D.L., y Aderson, R.D. (1986). Macho personality, sexual aggression, and reactions to guided imagery of realistic rape. Journal of Research in Personality, 20, 77-94.
Observatorio Centroamericano sobre Violencia (2007). Denuncias de Violencia Intrafamiliar registradas por año y por fuente en El Salvador periodo 2001 a Agosto 2007. Recuperado el 17 de octubre 2007, de http://www.ocavi.com/docs_files/file_325.pdf

Orantes, B.R. (2004). La situación de la mujer salvadoreña en el marco de la teoría de género. Enfoque jurídico-social. San Salvador: Universidad Tecnológica de El Salvador.

Reynolds, W.M. (1982). Development of reliable and valid short forms of the Marlowe-Crowne Scale of Social Desirability. Journal of Clinical Psychology, 38, 119125.

Ruch, L.O., y Wang, C. (2006). Validation of the Sexual Assault Symptom Scale II (SASS II) using a panel research design. Journal of Interpersonal Violence, 21, 1440-1461.

Ruiz-Pérez, I., Mata-Pariente, N., y Plazaola-Castaño, J. (2006). Women's response to intimate partner violence. Journal of Interpersonal Violence, 21, 1156-1168.

Sierra, J.C., y Gutiérrez-Quintanilla, R. (2007). Validación de la versión española del Cuestionario de Agresión de Buss-Perry en estudiantes universitarios salvadoreños. Psicología y Salud, 17, 103-113.

Sierra, J.C., y Gutiérrez-Quintanilla, R. (en prensa). Estudio psicométrico de la versión salvadoreña de la Double Standard Scale. Cuadernos de Medicina Psicosomática y Psiquiatría de Enlace.

Sierra, J.C., Gutiérrez-Quintanilla, R., y Delgado-Domínguez, C.J. (en prensa). Escala de Actitud Favorable hacia la Violación: primeras evidencias acerca de su fiabilidad y validez en muestras salvadoreñas. Universitas Psychologica.

Sipsma, E., Carrobles Isabel, J.A., Montorio Cerrato, I., y Everaerd, W. (2000). Sexual aggression against women by men acquaintances: Attitudes and experiences among Spanish university students. Spanish Journal of Psychology, 3, 14-27.

Strahan, R., y Gerbasi, K.C. (1972). Short, homogeneous versions of the Marlowe-Crowne Social Desirability Scale. Journal of Clinical Psychology, 28, 191-193.

Sugarman, D.B., y Hotaling, G.T. (1997). Intimate violence and social desirability. A meta-analytic review. Journal of Interpersonal Violence, 12, 275-290.

Ullman, S.E., Filipas, H.H., Townsend, S.M., y Starzynski, L.L. (2006). The role of victim-offender relationship in women's sexual assault experiences. Journal of Interpersonal Violence, 21, 798-819.

Waszak Geary, C., Wedderburn, M., McCarraher, D., Cuthberston, C., y Pottinger, A. (2006). Sexual violence and reproductive health among young people in three communities in Jamaica. Journal of Interpersonal Violence, 21, 1512-1533. 
Anexo 1.Versión española del Aggressive Sexual Behavior Inventory de Mosher y Anderson (1986)

Las siguientes son muestras de comportamiento que en ocasiones ocurren en las citas o en la interacción socio-sexual del hombre y la mujer. Cada ítem es contestado en una escala de frecuencia desde 1 (nunca) a 7 (siempre). Por favor, señale a la derecha de cada pregunta el número que mejor indique su comportamiento.

1 He amenazado con abandonar o terminar una relación si una mujer no tenía sexo conmigo.

2 He emborrachado a una mujer para tener sexo con ella.

3 He esperado mi turno entre otros hombres que estaban compartiendo a una «chica de la vida alegre».

4 Le he dicho a una mujer que he querido entrar a su departamento y así poder hacerle el amor donde yo quisiera.

5 Para que una mujer se tranquilice y disfrute le diría que podría lastimarla si se resiste

6 He drogado a una mujer con marihuana o pastillas para que pudiera resistirse menos a mis forcejeos.

7 Le he dicho a una mujer que por haberla estado acariciando tanto, no me podía dejar excitado.

8 He perdido los estribos y he quebrado algo para mostrarle a una mujer que no debía enojarme.

9 He traído a mi casa a una mujer después de una cita y la he obligado a tener sexo conmigo.

10 Le he dicho a una mujer que yo tenía citas para encontrar a alguien más para tener sexo si ella no lo hacía.

11 He tranquilizado a una mujer con una o dos buenas bofetadas al ponerse histérica por mis forcejeos.

12 Le he prometido a una mujer que no le haría daño si ella hacía todo lo que le dijera.

13 He ofendido a una mujer y la he empujado al no acceder a mi necesidad de sexo.

14 He obligado a una mujer a tener sexo conmigo y con algunos amigos.

15 Le he dado una droga cara a una mujer para que se sintiera obligada a hacerme un favor sexual.

16 He tratado mal a una mujer para que supiera que hablaba en serio.

17 He acostado a la fuerza a una mujer y la he hecho desvestirse o he roto su ropa si no cooperaba.

18 He tomado a una mujer fuertemente y la he visto muy enojado cuando no me respondía sexualmente como yo quería.

19 Me he emborrachado un poco y he obligado a la mujer con quien estoy a tener sexo conmigo.

20 Le he dicho a una mujer que su rechazo a tener sexo conmigo estaba cambiando mis sentimientos hacia ella.

\begin{tabular}{|c|c|c|c|c|c|}
\hline 1 & 2 & 3 & 4 & 5 & 6 \\
\hline 1 & 2 & 3 & 4 & 5 & 6 \\
\hline 1 & 2 & 3 & 4 & 5 & 6 \\
\hline 1 & 2 & 3 & 4 & 5 & 6 \\
\hline 1 & 2 & 3 & 4 & 5 & 6 \\
\hline 1 & 2 & 3 & 4 & 5 & 6 \\
\hline 1 & 2 & 3 & 4 & 5 & 6 \\
\hline 1 & 2 & 3 & 4 & 5 & 6 \\
\hline 1 & 2 & 3 & 4 & 5 & 6 \\
\hline 1 & 2 & 3 & 4 & 5 & 6 \\
\hline 1 & 2 & 3 & 4 & 5 & 6 \\
\hline 1 & 2 & 3 & 4 & 5 & 6 \\
\hline 1 & 2 & 3 & 4 & 5 & 6 \\
\hline 1 & 2 & 3 & 4 & 5 & 6 \\
\hline 1 & 2 & 3 & 4 & 5 & 6 \\
\hline 1 & 2 & 3 & 4 & 5 & 6 \\
\hline 1 & 2 & 3 & 4 & 5 & 6 \\
\hline 1 & 2 & 3 & 4 & 5 & 6 \\
\hline 1 & 2 & 3 & 4 & 5 & 6 \\
\hline 1 & 2 & 3 & 4 & & 6 \\
\hline
\end{tabular}

